NASA Technical Memorandum 106378

ICOMP-93-40; CMOTT-93-14

\title{
Computation of Confined Coflow Jets With Three Turbulence Models
}

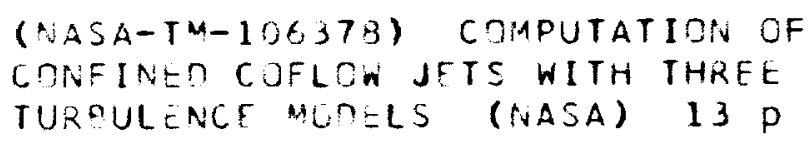

Unclas

$63 / 34 \quad 0193168$

\section{J. Zhu and T.H. Shih}

\section{Institute for Computational Mechanics in Propulsion} and Center for Modeling of Turbulence and Transition

Lewis Research Center

Cleveland, Ohio

Prepared for the

24th AIAA Fluid Dynamics Conference

sponsored by the American Institute of Aeronautics and Astronautics Orlando, Florida, July 6-9, 1993

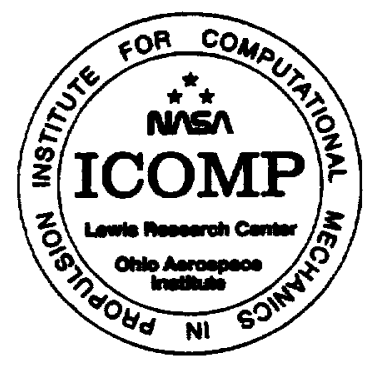


. 


\title{
COMPUTATION OF CONFINED COFLOW JETS WITH THREE TURBULENCE MODELS
}

\author{
J. Zhu and T.H. Shih \\ Institute for Computational Mechanics in Propulsion \\ and Center for Modeling of Turbulence and Transition \\ Lewis Research Center \\ Cleveland, Ohio 44135
}

\begin{abstract}
A numerical study of confined jets in a cylindrical duct is carried out to examine the performance of two recently proposed turbulence models: an RNG-based $K-\epsilon$ model and a realizable Reynolds stress algebraic equation model. The former is of the same form as the standard $K-\epsilon$ model but has different model coefficients. The latter uses an explicit quadratic stressstrain relationship to model the turbulent stresses and is capable of ensuring the positivity of each turbulent normal stress. The flow considered involves recirculation with unfixed separation and reattachment points and severe adverse pressure gradients, thereby providing a valuable test of the predictive capability of the models for complex flows. Calculations are performed with a finite-volume procedure. Numerical credibility of the solutions is ensured by using second-order accurate differencing schemes and sufficiently fine grids. Calculations with the standard $K-\epsilon$ model are also made for comparison. Detailed comparisons with experiments show that the realizable Reynolds stress algebraic equation model consistently works better than does the standard $K-\epsilon$ model in capturing the essential flow features, while the RNG-based $K-\epsilon$ model does not seem to give improvements over the standard $K-\epsilon$ model under the flow conditions considered.
\end{abstract}

\section{Introduction}

The flow configuration considered in this paper is sketched in Fig.1. It involves an inner high speed round jet and a slowly moving annular stream, both interacting with each other. Because of turbulent entrainment, the jet increases its mass fiux while spreading. This must be balanced by an equal decrease in the mass flux of the ambient flow. The decrease in the ambient velocity thus sets up an adverse pressure gradient which affects, in turn, the evolution of the flow. Depending on the ratio of jet to ambient velocities at the entrance, two different flow regimes occur in the downstream region: if the ratio is small, the jet cannot consume all the ambient flow before reaching the duct wall so that the flow remains unseparated; if the ratio is large, the opposite happens and further entrainment must create reverse flow to maintain the total mass flux conservation.
Further downstream, the flow completely loses its jet characteristics and degenerates eventually to the fully developed regime if the duct is long enough. These flow features can be found in many engineering apparatuses involving two flows of differing velocities, in particular, in combustion chambers and ejectors. Therefore, the understanding of confined jet flows is of great interest in engineering applications.

From a turbulence modeling point of view, the confined jet flow also constitutes a valuable test due to its complicated flow features. It is noted that the flow past a backward-facing step is a standard test problem to benchmark the performance of turbulence models in complex flows. The confined jet flow has features similar to those found in the backward-facing step flow such as recirculation with an unfixed reattachment point and severe adverse pressure gradient, and adds additional complexities arising from the motion of the separation point.

Numerical calculations of confined jets have been reported by Gosman et al. ${ }^{1}$, Habib and Whitelaw ${ }^{2,3}$, Jones and Marquis, ${ }^{4}$ Khalil et al. ${ }^{5}$ and $\mathrm{Zhu}^{6}$. In these calculations, turbulence effects were represented either by the $K-\epsilon$ model or by second-moment closures. However, the previous calculations were all made on very coarse grids and with the hybrid central/upwind scheme $^{7}$ that is highly diffusive in the presence of both convective dominance and flow-to-grid skewness. Therefore, they might largely be contaminated by numerical diffusion, and the results are far from conclusive.

The purpose of the present study is to assess the performance of two recently developed turbulence models in the confined jet flow. The models considered here are the RNG-based $K-\epsilon$ model used by Speziale and Thangam $^{8}$ and the realizable Reynolds stress algebraic equation (RRSAE) model ${ }^{\theta}$, both within the framework of the two-equation formulation. The RNG-based $K-\epsilon$ model is of the same form as the standard $K-\epsilon$ model $^{10}$ but assumes different model coefficients which are evaluated by the theory. In the original version of the RNG $K-\epsilon$ model, all the coefficients had constant values which have been shown by Speziale and Thangam ${ }^{8}$ to be inappropriate. In the latest version of the RNG $K-\epsilon$ model ${ }^{8}$, the model coefficient related to the production of dissipation term in the $\epsilon$ equation is a function of 
$\eta$, where $\eta$ is the time scale ratio of the turbulent to mean strain rate. In the RRSAE model, the Reynolds stresses are calculated by a quadratic stress-strain relation. All the model coefficients in this relation are determined from the realizability analysis so that the model ensures the positiveness of the turbulent normal stresses.

The test problem to be considered is taken from the experiment of Barchilon and Curtet ${ }^{11,12}$ which provides detailed experimental data. The flow can be characterized by the Craya-Curtet number $C_{t}$ which is the inverse square root of the total momentum, nondimensionalized with the volume flux and the duet area ${ }^{1}$. The experiment showed that recirculation occurs when $C_{t} \leq 0.96$. Calculations are carried out with a conservative finite-volume method and on a numerically accurate basis. As a common practice, the calculation with the standard $K-\epsilon$ model is also included for comparison. Detailed comparisons with experiments at five $C_{t}$ numbers clearly reveal the predictive capabilities of the models in these flows of great practical importance.

\section{Mathematical Formulation}

\subsection{Governing Equations}

Incompressible, steady-state, turbulent flows are governed by the Reynolds-averaged continuity and Navier-Stokes equations. In the polar-cylindrical coordinate system $(x, r)$ shown in Fig.1, the conservative form of these equations can be written as

$$
\begin{gathered}
\frac{\partial r U}{\partial x}+\frac{\partial r V}{\partial r}=0 \\
\frac{\partial}{\partial x}\left[r\left(U U-\nu \frac{\partial U}{\partial x}\right)\right]+\frac{\partial}{\partial r}\left[r\left(V U-\nu \frac{\partial U}{\partial r}\right)\right] \\
=-\frac{r}{\rho} \frac{\partial p}{\partial x}+\frac{\partial}{\partial x}\left[r\left(\nu \frac{\partial U}{\partial x}+\frac{\tau_{11}}{\rho}\right)\right] \\
+\frac{\partial}{\partial r}\left[r\left(\nu \frac{\partial V}{\partial x}+\frac{\tau_{12}}{\rho}\right)\right] \\
\frac{\partial}{\partial x}\left[r\left(U V-\nu \frac{\partial V}{\partial x}\right)\right]+\frac{\partial}{\partial r}\left[r\left(V V-\nu \frac{\partial V}{\partial r}\right)\right] \\
=-\frac{r}{\rho} \frac{\partial p}{\partial r}+\frac{\partial}{\partial x}\left[r\left(\nu \frac{\partial U}{\partial r}+\frac{\tau_{12}}{\rho}\right)\right] \\
+\frac{\partial}{\partial r}\left[r\left(\nu \frac{\partial V}{\partial r}+\frac{\tau_{22}}{\rho}\right)\right]-\frac{2 \nu V}{r}-\frac{\tau_{33}}{\rho}
\end{gathered}
$$

where $U$ and $V$ are the axial and radial velocities, respectively, $p$ is the pressure, $\nu$ is the kinematic viscosity and $\rho$ is the density. The Reynolds stresses $\tau_{i j}$ in Eqs.( 2) and (3) are calculated by using the following three turbulence models:
1) Standard $K-\epsilon$ (SKE) model ${ }^{10}$

$$
\begin{gathered}
\frac{\tau_{i j}}{\rho}=\nu_{t}\left(U_{i, j}+U_{j, i}\right)-\frac{2}{3} K \delta_{i j}, \quad \nu_{t}=C_{\mu} \frac{K^{2}}{\epsilon} \\
\frac{\partial}{\partial x}\left\{r\left[U K-\left(\nu+\frac{\nu_{t}}{\sigma_{K}}\right) \frac{\partial K}{\partial x}\right]\right\}+\frac{\partial}{\partial r}\left\{r\left[V K-\left(\nu+\frac{\nu_{t}}{\sigma_{K}}\right) \frac{\partial K}{\partial r}\right]\right\} \\
=G-\epsilon \\
\frac{\partial}{\partial x}\left\{r\left[U \epsilon-\left(\nu+\frac{\nu_{t}}{\sigma_{\epsilon}}\right) \frac{\partial \epsilon}{\partial x}\right]\right\}+\frac{\partial}{\partial r}\left\{r\left[V \epsilon-\left(\nu+\frac{\nu_{t}}{\sigma_{\epsilon}}\right) \frac{\partial \epsilon}{\partial r}\right]\right\} \\
=C_{1} G \frac{\epsilon}{K}-C_{2} \frac{\epsilon^{2}}{K}
\end{gathered}
$$

where $G$ is the production term of the turbulent kinetic energy

$$
G=\frac{1}{\rho}\left[\tau_{11} U_{1,1}+\tau_{22} U_{2,2}+\tau_{33} U_{3,3}+\tau_{12}\left(U_{1,2}+U_{2,1}\right)\right]
$$

the velocity gradients $U_{i, j}$ are calculated by

$$
\begin{aligned}
& U_{1,1}=\frac{\partial U}{\partial x}, \quad U_{2,2}=\frac{\partial V}{\partial r}, \quad U_{3,3}=\frac{V}{r}, \\
& U_{1,2}=\frac{\partial U}{\partial r}, \quad U_{2,1}=\frac{\partial V}{\partial x}
\end{aligned}
$$

and the model coefficients are

$$
\begin{aligned}
& C_{\mu}=0.09, \quad C_{1}=1.44, \quad C_{2}=1.92, \\
& \sigma_{K}=1, \quad \sigma_{\epsilon}=1.3
\end{aligned}
$$

2) RNG $K-\epsilon$ model $^{8}$. It is of the same form as the standard $K-\epsilon$ model but uses the following coefficients

$$
\begin{aligned}
& C_{\mu}=0.085, C_{1}=1.42-\frac{\eta(1-\eta / 4.38)}{1+0.015 \eta^{3}}, \\
& C_{2}=1.68, \sigma_{K}=\sigma_{\epsilon}=0.7179
\end{aligned}
$$

where

$$
\eta=\frac{K S}{\epsilon}, S=\left(2 S_{i j} S_{i j}\right)^{1 / 2}, S_{i j}=\frac{1}{2}\left(U_{i, j}+U_{j, i}\right)
$$

3) RRSAE model ${ }^{\theta}$

$$
\frac{\tau_{i j}}{\rho}=\nu_{t}\left(U_{i, j}+U_{j, i}\right)+T_{i j}-\frac{2}{3} K \delta_{i j}, \quad \nu_{t}=C_{\mu} \frac{K^{2}}{\epsilon}
$$

where

$$
\begin{gathered}
C_{\mu}=\frac{2 / 3}{A_{1}+\eta+\gamma \xi} \\
T_{i j}=-\frac{K^{3}}{\left(A_{2}+\eta^{3}\right) \epsilon^{2}}\left[C_{\tau 1}\left(U_{i, k} U_{k, j}+U_{j, k} U_{k, i}-\frac{2}{3} \Pi \delta_{i j}\right)\right. \\
\left.+C_{\tau 2}\left(U_{i, k} U_{j, k}-\frac{1}{3} \bar{\Pi} \delta_{i j}\right)+C_{\tau 3}\left(U_{k, i} U_{k, j}-\frac{1}{3} \tilde{\Pi} \delta_{i j}\right)\right]
\end{gathered}
$$




$$
\begin{aligned}
& \Pi=U_{k, l} U_{l, k}, \quad \tilde{\Pi}=U_{k, l} U_{k, l} \\
& \xi=\frac{K \Omega}{\epsilon}, \quad \Omega=\left(2 \Omega_{i j}^{*} \Omega_{i j}^{*}\right)^{1 / 2}, \\
& \Omega_{i j}^{*}=\left(U_{i, j}-U_{j, i}\right) / 2+4 \epsilon_{m j i} \omega_{m}
\end{aligned}
$$

$\omega_{m}$ is the rotation rate of the reference frame, and the model constants are

$$
C_{\tau 1}=-4, C_{\tau 2}=13, C_{\tau 3}=-2, A_{2}=1000 \text {. }
$$

In the work of Shih et al. ${ }^{8}$ and Zhu and Shih ${ }^{14}$, the following two sets of values for $A_{1}, \gamma$ were tested

$$
\begin{gathered}
A_{1}=5.5, \quad \gamma=0 \\
A_{1}=1.25, \quad \gamma=0.9
\end{gathered}
$$

and both of them have been found to give almost identical predictions for the two backward-facing step flows. With Eq.(19), the rotational effect of the mean flow enters into $C_{\mu}$. However, we have found in the present work that the values in Eq.( 18) work better for the axisymmetric confined jets. Therefore the values in Eq. (18) are taken here. The $K$ and $\epsilon$ in the RRSAE model are calculated with the same equations as in the standard $K$ - $\epsilon$ model.

\subsection{Boundary Conditions}

Four types of boundaries are present in the calculation; they are the inlet, outlet, axis of symmetry and solid wall. Among them, the inlet boundary conditions demand special attention because they have a considerable influence on the calculations ${ }^{15,16}$. Table 1 gives the inlet jet and ambient velocities taken from the experiment of Barchilon and Curtet ${ }^{11}$

\begin{tabular}{ccc}
\multicolumn{3}{c}{ Table 1. Inflow conditions } \\
\hline \hline$C_{t}$ & $U_{J}(\mathrm{~cm} / \mathrm{s})$ & $U_{a}(\mathrm{~cm} / \mathrm{s})$ \\
\hline 0.976 & 1293.6 & 84.48 \\
\hline 0.714 & 1298.9 & 60.72 \\
\hline 0.506 & 1253.8 & 39.81 \\
\hline 0.305 & 1282.1 & 21.86 \\
\hline 0.152 & 1296.2 & 7.42 \\
\hline
\end{tabular}

The Craya-Curtet number $C_{t}$ is calculated by

$$
C_{t}=\frac{U_{m}}{\left[\left(U_{J}^{2}-U_{a}^{2}\right)\left(d_{o} / D_{o}\right)^{2}+\left(U_{\alpha}^{2}-U_{m}^{2}\right) / 2\right]^{1 / 2}}
$$

where $D_{o}=16 \mathrm{~cm}, d_{o}=1.2 \mathrm{~cm}$ and $U_{m}$ is the mean velocity of the section

$$
U_{m}=\left(U_{J}-U_{a}\right)\left(d_{o} / D_{0}\right)^{2}+U_{a}
$$

In the potential core and the ambient region (Fig.1), the velocity are uniform and the turbulence level is very low so that the flow may be treated as potential. However, between the potential core and the ambient region there exists a thin shear layer from which the turbulent entrainment develops. The specification of the boundary conditions in this layer is nontrivial. In this work, the parabolic entrance region (PER) scheme of Zhu et al. ${ }^{15}$ is used. The PER scheme which allows the fine resolution of the initial shear layer was developed on the assumption that although the flow as a whole is elliptic, there exists a short region near the entrance where the flow is parabolic. A parabolic calculation is first carried out over a short distance between $x=0$ and $x=x_{e}$, by using the following mixing length model

$$
\overline{u v}=-\nu_{t} \frac{\partial U}{\partial r}, \quad \nu_{t}=C^{2}\left(r_{2}-r_{1}\right)^{2}\left|\frac{\partial U}{\partial r}\right|
$$

where $r_{1}$ and $r_{2}$ are the coordinates of the inner and onter edge of the initial shear layer (Fig.1) and $C$ is an empirical coefficient given by

$$
C^{2}=0.0042+0.004 U_{a} / U_{J}, \quad 0 \leq U_{a} / U_{J} \leq 0.2
$$

The results of the parabolic calculation are then used as the inlet conditions at $x=x_{e}$ for the elliptic calculation. The inlet values of $K$ and $\epsilon$ are calculated by

$$
K=-\overline{u v} / 0.3, \quad \epsilon=0.09 K^{2} / \nu_{t}
$$

It was found ${ }^{6}$ that the PER scheme gives satisfactory predictions in the parabolic entrance region and the elliptic calculations were insensitive to $x_{e}$ provided that $1<x_{e} / d_{0}<3$.

The outlet boundary is placed at $x=10 D_{0}$ where fully-developed flow conditions are assumed. Along the axis of symmetry the normal velocity component and the normal gradients of the other variables are set to zero. The standard wall-function approach ${ }^{10}$ is used to handle the wall boundary conditions.

\subsection{Numerical Procedure}

The transport equations ( 1$),(2),(3),(5)$ and (6) can be written in the following general form

$$
\frac{\partial}{\partial x}\left[r\left(U \phi-\Gamma_{\phi} \frac{\partial \phi}{\partial x}\right)\right]+\frac{\partial}{\partial r}\left[r\left(V \phi-\Gamma_{\phi} \frac{\partial \phi}{\partial r}\right)\right]=S_{\phi}
$$

where $\phi$ stands for $U, V, K$ and $\epsilon$, and $\Gamma_{\phi}$ and $S_{\phi}$ are the corresponding diffusive coefficient and source term, respectively. For the momentum equations ( 2) and $(3), S_{\phi}$ also includes the cross-derivative diffusion terms and the quadratic terms $T_{i j}$ in Eq. (14).

The system of equations ( 25 ) is solved with the finite-volume approach. It uses non-staggered grids with all the dependent variables being stored at the geometric center of each control volume. The velocitypressure coupling is handled with the momentum interpolation procedure of Rhie and $\mathrm{Chow}^{17}$ and the SIMPLEC algorithm of Van Doormal and Raithby ${ }^{18}$. 
To ensure both accuracy and stability of numerical solutions, the hybrid linear/parabolic approximation (BLPA) scheme ${ }^{19}$ is used to approximate the convection terms of Eq.( 25). It has been shown ${ }^{20}$ that the HLPA scheme of second-order accuracy works nearly as well as the third-order accurate SMART ${ }^{21}$ and SHARP ${ }^{22}$ schemes in eliminating the numerical diffusion while retaining the boundedness of numerical solutions. Considering a typical control volume centered at node $\mathrm{C}$ shown in Fig.2. The HLPA scheme evaluates the value of $\phi$ at the cell-face $w$ as follows

$$
\phi_{w}=U_{w}^{+} \phi_{W}+U_{w}^{-} \phi_{C}+\Delta \phi_{w}
$$

where

$$
\begin{gathered}
\Delta \phi_{w}=U_{w}^{+} \alpha_{w}^{+}\left(\phi_{C}-\phi_{W}\right) \frac{\phi_{W}-\phi_{W W}}{\phi_{C}-\phi_{W W}} \\
+\quad U_{w}^{-} \alpha_{w}^{-}\left(\phi_{W}-\phi_{C}\right) \frac{\phi_{C}-\phi_{E}}{\phi_{W}-\phi_{E}} \\
U_{w}^{+} \equiv \frac{1}{2}\left(1+\frac{\left|U_{w}\right|}{U_{w}}\right), \quad U_{w}^{-} \equiv 1-U_{w}^{+} \quad\left(U_{w} \neq 0\right) \\
\alpha_{w}^{+}= \begin{cases}1 & \text { if }\left|\phi_{C}-2 \phi_{W}+\phi_{W W}\right|<\left|\phi_{C}-\phi_{W W}\right| \\
0 & \text { otherwise }\end{cases} \\
\alpha_{w}^{-}= \begin{cases}1 & \text { if }\left|\phi_{W}-2 \phi_{C}+\phi_{E}\right|<\left|\phi_{W}-\phi_{E}\right| \\
0 & \text { otherwise }\end{cases}
\end{gathered}
$$

It can be seen that Eq. (26) is in fact the result of the first-order upwinding $U_{w}^{+} \phi_{W}+U_{w}^{-} \phi_{C}$ with an additional term $\Delta \phi_{w}$ added. The additional term may be viewed as an antidiffusive correction to the upwind scheme. The conventional central differencing scheme is used to approximate all other terms. The resulting discretized counterpart of Eq.( 25) can be cast into the following linearized form:

$$
A_{C} \phi_{C}=A_{i} \phi_{i}+S, \quad i=W, E, S, N
$$

In formulating this equation, the convection terms calculated by the upwind scheme are coupled with the normal diffusion terms to form the main coefficients $A_{i}$, while those calculated by Eq.( 27) are included in the source term $S$. This way, the positivity of all the main coefficients is ensured so that the resulting coefficient matrix will be always diagonally dominant. The system of equations ( 31 ) is solved with the strongly implicit solution algorithm of Stone ${ }^{23}$. The calculation results are considered converged when the maximum normalised residue of all the dependent variables is less than $0.5 \%$. The details of the present numerical procedure are given in Rodi et al. ${ }^{24}$ and $\mathrm{Zhu}^{25}$.

\section{Application}

All calculations were performed on the Cray YMP computer. The grid-dependency of solutions was first examined by using two convection schemes, BLPA and HYBRID (central/upwind differencing), and three grids consisting of $50 \times 40$ (grid 1), $86 \times 50$ (grid 2) and $120 \times 80$ (grid 3) points, respectively. The HYBRID scheme that is highly diffusive in the presence of both convective dominance and flow-to-grid skewness has been used here mainly to highlight the importance of using higher-order accurate schemes. Test results obtained with the RRSAE model at $C_{t}=0.506$ are shown in Fig.3(a) for the axial velocity $U$-profiles, normalized by the mean velocity of the section $U_{m}$, and in Fig.3(b) for the turbulent shear stress $\overline{u v}$-profiles, both at the same downstream location $x / D_{o}=1.875$. It can be seen that the results of BLPA on the coarse grid 1 are already very close to those on the fine grid 3 for both the $U$ - and the $\overline{u v}$-profiles, while significant differences exist between the corresponding results of HYBRID. The HLPA results on the intermediate grid 2 can be considered as grid-independent because the refinement from the grid 2 to the grid 3 produced differences too small to be seen on the graph. The HYBRID solutions, however, responded to the grid refinement in such a slow manner that they still had not reached the grid-independent stage on the finest grid. The numbers of iterations and CPU-time in minutes required for the calculations with HLPA were 196 and 0.2 on grid 1,640 and 1.4 on grid 2 and 1874 and 9.3 on grid 3 . The calculations with HYBRID took about $0.6 \sim 0.8$ of these numbers. The grid 2 and HLPA were used for all subsequent calcualtions.

Fig.4 shows the variation of the centerline velocity $U_{o}$ with $x$ and $C_{t}$. It clearly reveals the existence of the potential core characterized by the constant $U_{0}$ in the near-entrance region. Beyond the potential core, $U_{0}$ decayed quickly, especially at small values of $C_{t}$. Both the SKE model and the RNG model predicted the same potential core length which was shorter than that predicted with the RRSAE model at all the $C_{t}$ numbers. Since this length cannot be precisely determined from the first and second experimental points at each value of $C_{t}$, it is difficult to judge which model gives the better initial decay $\left(x / D_{0}<1\right)$. For the ensuing decay, the RRSAE model gave the best agreement with the experiment while the RNG model produced large underpredictions. Figs.5(a)-(c) show the axial mean velocity profiles at three $C_{t}$ numbers. All the three models are seen to predict very well the upstream evolution of the flow. As for the downstream development, the results obtained with the RRSAE model remained in good agreement with experiments, while those obtained with the other two models deteriorated with the RNG model producing the largest discrepencies. The variation of the ambient velocity $U_{1}$ with $x$ and $C_{t}$ is shown in Fig.6. In the recirculation region, the ambient veloc- 
ity has no physical meaning and is defined as the minimum velocity (Fig.1) for analytical convenience. The location where $U_{1}$ is equal to zero corresponds to the separation or reattachment point. At $C_{t}=0.976$, the calculated results are shown only up to $x / D_{o}=1.875$ because the calculated $U$-profiles have no uniform portion after this point (Fig.5a). The calculated curves follow quite well with the experimental data upstream of the separation at all the $C_{t}$ numbers. The deviation occurs in the recirculation region. It should be pointed out that in the recirculation region, the computed velocity minimums are all very close to the duct wall where the use of the wall-function as the boundary condition may constitute a source of error. It is also to be noted that Fig. 6 highlights considerably the difference between the computed and measured $U$-profiles in the near-wall region. This difference shown in Figs.5(b) and 5(c) is not as significant as in Fig.6. Therefore, the RRSAE model result should be considered as satisfactory.

The jet spreading can be characterized by the excess flow rate $Q_{j}$ and the effective width $l$. They are defined by

$$
Q_{j}=2 \pi \int_{0}^{\tau_{2}}\left(U-U_{1}\right) r d r, \quad l^{2}=\frac{Q_{j}}{\pi\left(U_{o}-U_{1}\right)}
$$

Figs.7 and 8 show the variation of $Q_{j} / Q$ and $l / R$ with $x$ and $C_{t}$, where $Q$ and $R$ are the total flow rate and the radius of the duct. As a result of the turbulent entrainment, the excess flow rate increases first, reaches a maximum at the recirculation center where $U_{1}$ has a minimum and then decreases. This variation becomes more marked as $C_{t}$ decreases. Recirculation occurs when $Q_{j}$ is larger than $Q$. It can be seen from both figures that the calculations agree well with the experiments at larger $C_{t}$, but the agreement deteriorates as $C_{t}$ decreases. It should be pointed out that the excess flow rate, due to its definition, is a quantity that is highly sensitive to the errors in the velocity profiles so that a small change in $U_{1}$, especially in the recirculation zone, will result in a large difference in $Q_{j}$. Furthermore, the experimental uncertainty in the recirculation region in which the flow is highly perturbed is likely to be greatest. With due regard to these factors, the agreement between the calculations and experiments can be regarded as reasonably good. Regarding the comparison among the three models, the RRSAE model again performs the best for both the excess flow rate and the effective width.

Figs.9(a) and 9(b) show the predicted streamlines at the two values of $C_{t}=0.714$ and 0.152 . These figures convey an overall view of the flow pattern. The upstream ambient flow was sucked in by the jet due to the turbulent entrainment. At $C_{t}=0.714$, a small recirculating bubble adhering to the duct wall occurred at the downstream location. When $C_{t}$ was reduced to 0.152 , the recirculating bubble became very large, filling the most of the duct cross-section. The separation and reattachment points of the predicted recirculating bubbles are compared with the experimental data in Fig. 10. The experiment indicated that as $C_{t}$ decreased, the separation point moved upstream while the reattachment point remained practically unchanged. The comparison shows that the RRSAE model gives the best predictions for both the separation and reattachment points.

Fig.11 shows the variation of the recirculating flow rate with $x$ at $C_{t}=0.305$ and 0.152 . This is the integral of negative velocities in each cross-section. The experiment indicated that the recireulating flow rate at $C_{t}=0.152$ is about 3 times larger than that at $C_{t}=0.305$. The results of the RRSAE model are in good agreement with the experiment while those of the standard $K-\epsilon$ model and the RNG model have substantial discrepancies. As for the maximum recirculating flow rate which is a critical parameter to characterize the performance of combustion chambers, the RRSAE model gave the same result as the experimental data at $C_{t}=0.305$ and a $9 \%$ overprediction at $C_{t}=0.152$ while the other two models produced larger overpredictions. It should be pointed out that results from different measurements ${ }^{11}$ for this quantity showed considerable scatter at small $C_{t}$ numbers. The results of all the three models are within the experimental scatter.

The variation of the pressure coefficient $C_{p}$ along the duct wall is shown in Fig.12, where, $C_{p}$ is defined by

$$
C_{p}=\frac{\Delta p-\rho U_{a}^{2} / 2}{\rho U_{J}^{2} / 2}
$$

and $\Delta p$ is the pressure difference relative to the entrance. In the cylindrical duct, the evolution of the pressure is governed by the jet entrainment as well as the contraction and expansion of the flow caused by the recirculating bubble. The decrease in the ambient velocity (Fig.6) induced by the entrainment gives rise to an adverse pressure gradient, while the contraction of streamlines produces the opposite effect. These two mechanisms interact more intensely with each other as $C_{t}$ decreases, causing the pressure to vary little in the region upstream of the center of the recirculating bubble. In the downstream part of the recirculating bubble, the deceleration of the flow sets up an adverse pressure gradient the slope of which becomes steeper as $C_{t}$ decreases. Therefore, the ability to capture the location of the recirculation center will have a direct impact on the prediction of the pressure. By comparing Fig.12 and Fig.6, it can be seen that the three models capture the steep pressure gradients in the same way as they capture the ambient velocity minimums. However, for the total pressure rise, an important parameter to the designer of jet pump devices, all the three models are seen to give the same results which are in excellent agreement with the measurement. 


\section{Conclusions}

A numerical study has been performed to assess two recently proposed turbulence models for confined jet flows. In order for the calculation to reflect the real performance of the models, an effort has been made to reduce numerical errors arising from the inlet boundary condition and numerical discretization. The detailed comparison with the experiment definitively establishes the superiority of the RRSAE model over the standard $K-\epsilon$ model in so far as the confined jet problem is concerned. However, this is not true for the RNG model at all the values of $C_{t}$ considered.

\section{References}

1. Gosman, A.D., Khalil, E.E., and Whitelaw, J.H., "The calculation of two-dimensional turbulent recirculating flows," Turbulent Shear Flows 1, edited by F. Durst, B.E. Launder, F.W. Schmidt, and J.H. Whitelaw, Springer-Verlag, 1979, pp.237-255.

2. Habib, M.A., and Whitelaw, J.H., "Velocity characteristics of a confined coaxial jet," Journal of Fluids Engineering, Vol.101, 1979, pp.521-529.

3. Habib, M.A., and Whitelaw, J.H., "Calculations of confined coaxial-jet flows," Proceedings, Turbulent Shear Flows 3, University of California, Davis, 1981, Pp.5.20-5.26.

4. Jones, W.P., and Marquis, A.J., "Calculation of axisymmetric recirculating flows with a second order turbulent model," Proceedings, Turbulent Shear Flows 5, Cornell University, Ithaca, 1985, pp.20.1-20.6.

5. Khalil, E.E., Spalding, D.B., and Whitelaw, J.H., "The calculation of local flow properties in two dimensional furnaces," International Journal of Heat and Mass Transfer, Vol.18, 1975, pp.775-791.

6. Zhu, J., "Calcul des jets turbulents confinés avec recirculation," Doctoral dissertation, l'Institut National Polytechnique de Grenoble, France, 1986.

7. Spalding, D.B., "A novel finite difference formulation for differential expressions involving both first and second derivatives," International Journal for Numerical Methods in Engineering, Vol.4, 1972, pp.551-559.

8. Speziale, C.G., and Thangam, S., "Analysis of an RNG based turbulence model for separated flows," NASA CR-189600, 1992.

9. Shih, T.-H., Zhu, J., and Lumley, J.L., "A realizable Reynolds stress algebraic equation model," NASA TM 105993, 1993.

10. Launder, B.E., and Spalding, D.B., "The numerical computation of turbulent flows," Computer Methods in Applied Mechanics and Engineering, Vol.3, 1974, pp.269-289.

11. Barchilon, M., and Curtet, R., "Some details of the structure of an axisymmetric confined jet with backlow," Journal of Basic Engineering, Vol.86, 1964, pp.777-787.

12. Barchilon, M., "Contribution à l'étude des courants de retour engendrés par un jet confiné," Publications Scientifiques et Techniques du Ministère de l'Air, Paris, 1969.
13. Curtet, R., "Sur l'écoulement d'un jet entre parois," Publications Scientifiques et Techniques du Ministère de l'Air, No.359, Paris, 1960.

14. Zhu, J., and Shih, T.-H., "Calculations of turbulent separated flows," NASA TM 106154, 1993.

15. Zhu, J., Binder, G., and Kueny, J.L., "Improved predictions of confined jets with a parabolic computation of the entrance region," AIAA Journal, Vol.25, 1987, pp.1141-1142.

16. Zhu, J., and Rodi, W., "Computation of axisymmetric confined jets in a diffuser," International Journal for Numerical Methods in Fluids, Vol.14, 1992, pp.241-251.

17. Rhie, C.M., and Chow, W.L., "A numerical study of the turbulent flow past an isolated airfoil with trailing edge separation," AIAA Journal, Vol.21, 1983, pp.1525-1532.

18. Van Doormal, J.P., and Raithby, G.D., "Enhancements of the SIMPLE method for predicting incompressible fluid flows," Numerical Heat Transfer, Vol.7, 1984, pp.147-163.

19. Zhu, J., "A low diffusive and oscillation-free convection scheme," Communications in Applied $\mathrm{Nu}$ merical Methods., Vol.7, 1991, pp.225-232.

20. Zhu, J., "On the higher-order bounded discretization schemes for finite-volume computations of incompressible filows," Computer Methods in Applied Mechanics and Engineering, Vol.98, 1992, pp.345360.

21. Gaskell, P.H., and Lau, A.K.C., "Curvaturecompensated convective transport: SMART, A new boundedness preserving transport algorithm," International Journal for Numerical Methods in Fluids, Vol.8, 1988, pp.617-641.

22. Leonard, B.P., "Simple high-accuracy resolution program for convective modelling of discontinuities," International Journal for Numerical Methods in Fluids, Vol.8, 1988, pp.1291-1318.

23. Stone, H.L., "Iterative solution of implicit approximations of multidimensional partial differential equations," SIAM J. Num. Anal, Vol.5, 1968, pp.530-558.

24. Rodi, W., Majumdar, S., and Schönung, B., "Finite-volume method for two-dimensional incompressible flows with complex boundaries," Computer Methods in Applied Mechanics and Engineering, Vol.75, 1989, pp.369-392.

25. Zhu, J., "FAST-2D: A computer program for numerical simulation of two-dimensional incompressible flows with complex boundaries," Rept. No.690, Institute for Bydromechanics, University of Karlsruhe, 1991. 


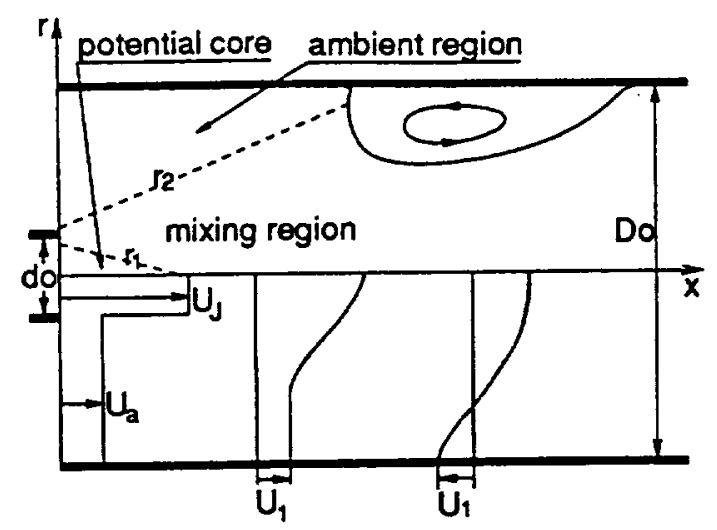

Fig.1 Flow configuration and notations

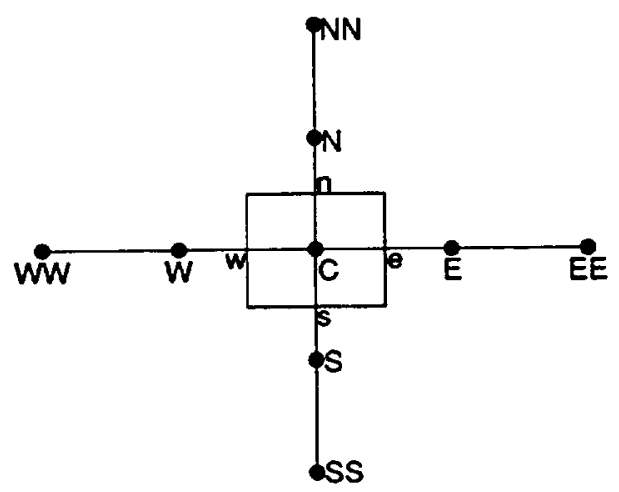

Fig.2 Control volume and related nodes

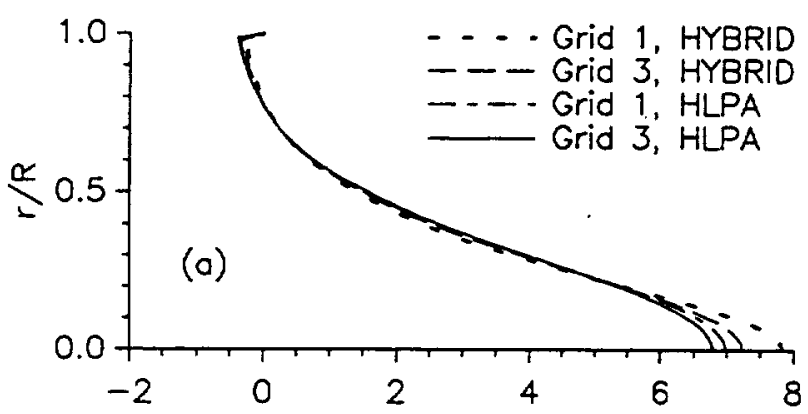

$U / U m$

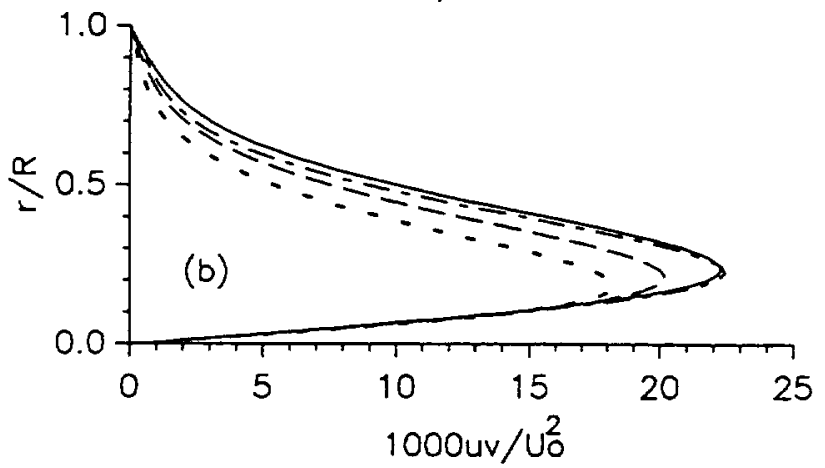

Fig.3 Grid sensitivity test at $C_{t}=0.506$

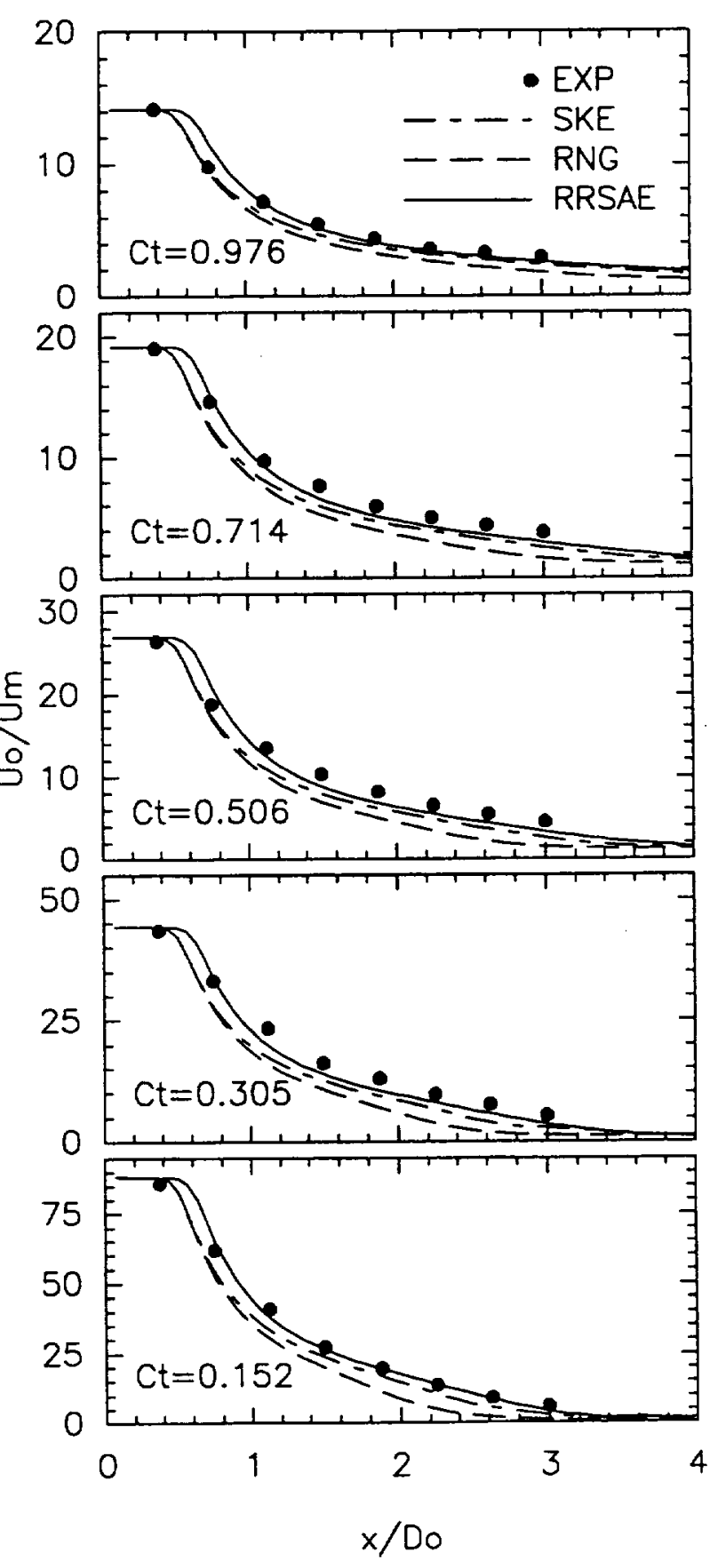

Fig.4 Centerline velocity decay 

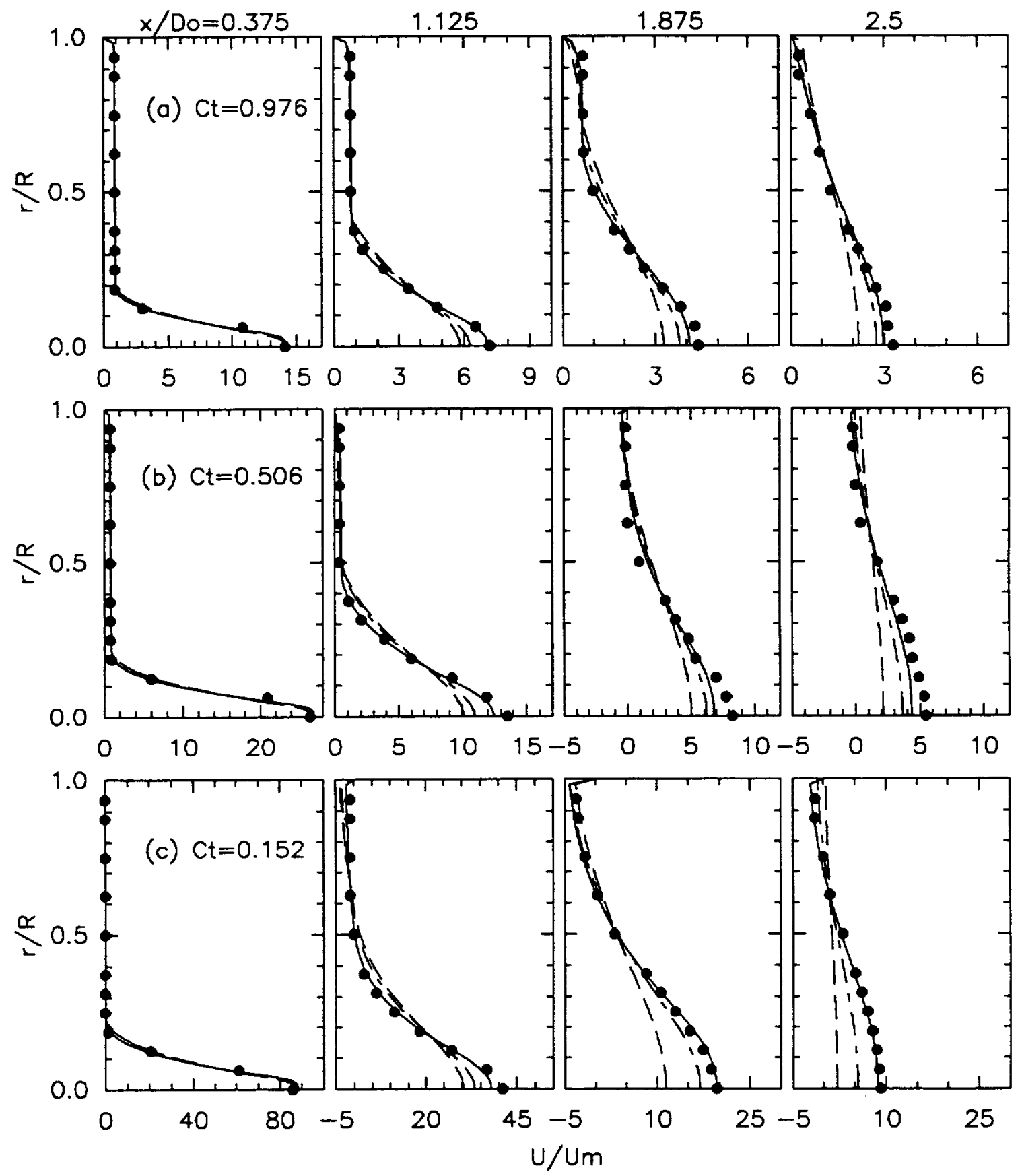

Fig.5 Axial mean velocity profiles (Notation as in Fig.4) 


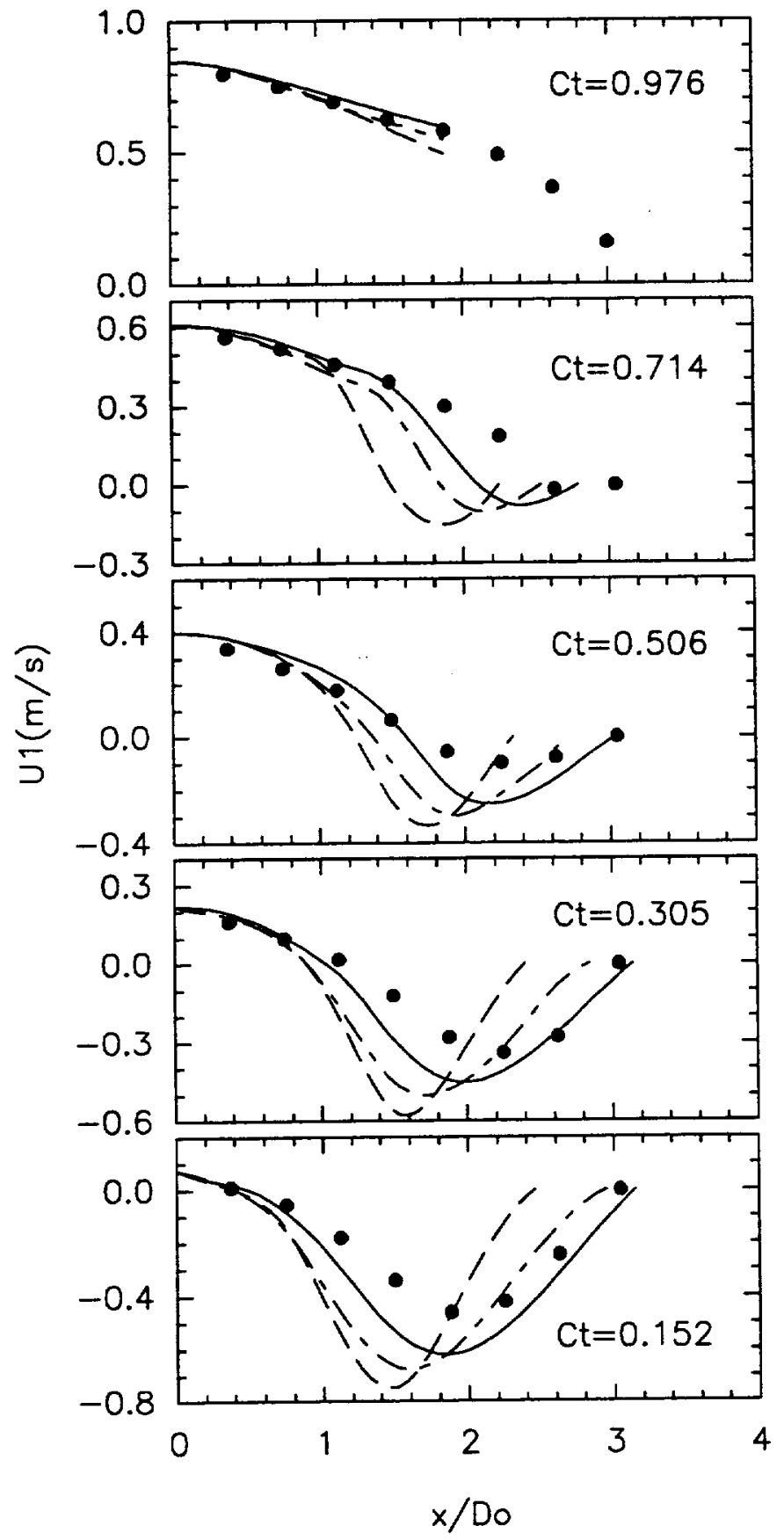

Fig.6 Ambient velocity (Notation as in Fig.4)

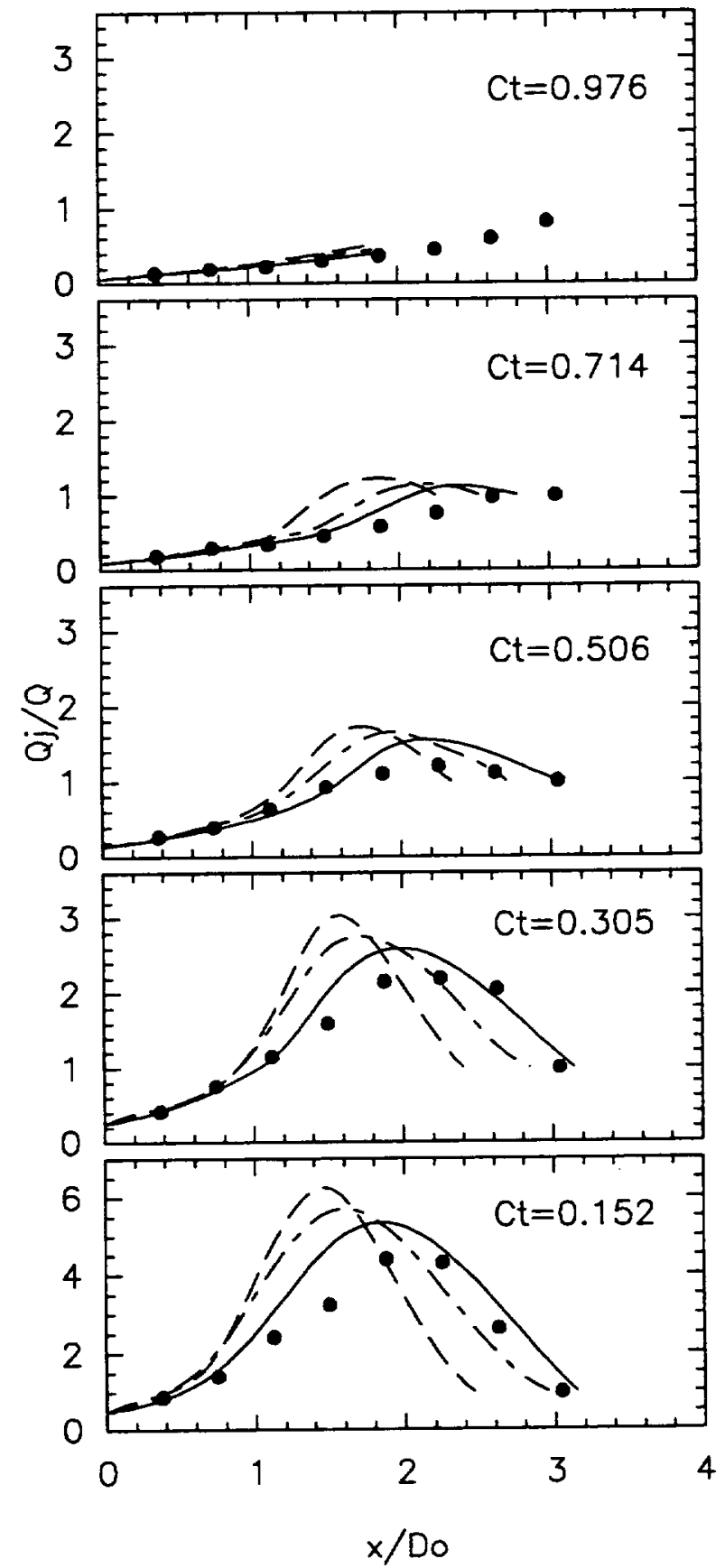

Fig.7 Excess flow rate (Notation as in Fig.4) 


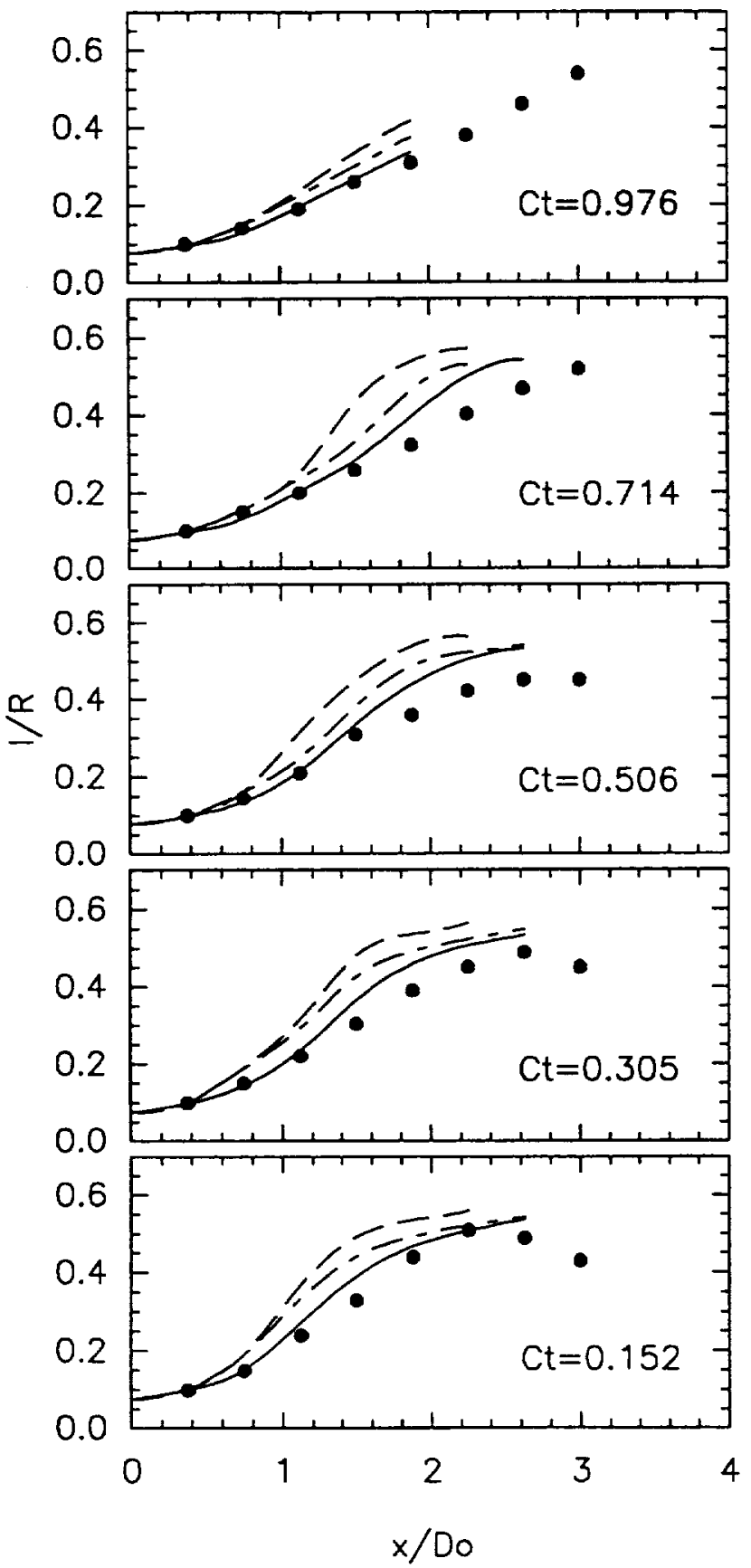

Fig.8 Effective width (Notation as in Fig.4)

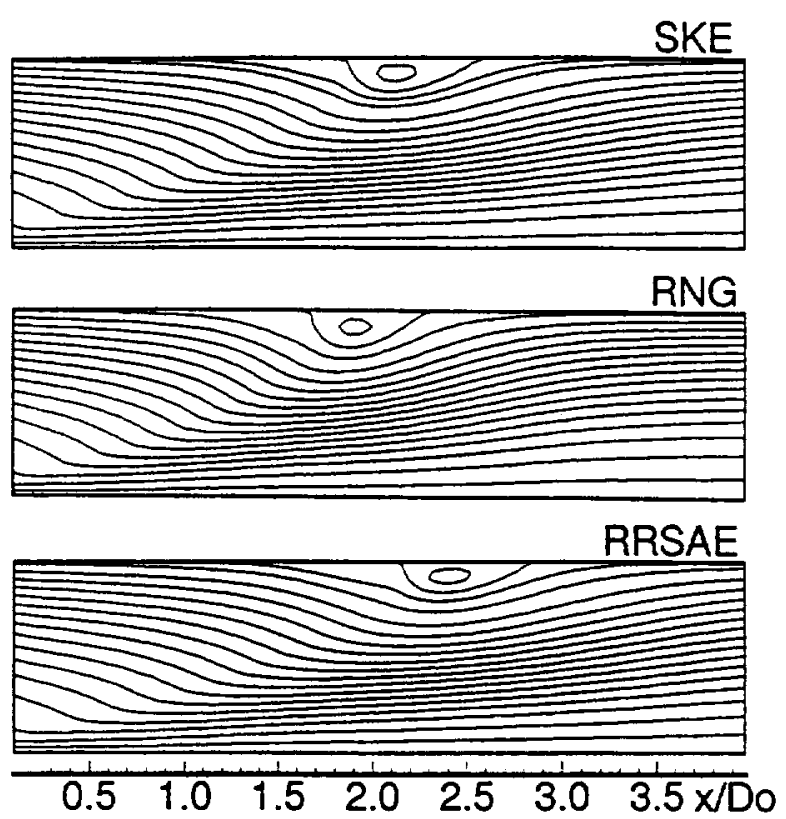

(a) $\mathrm{Ct}=0.714$

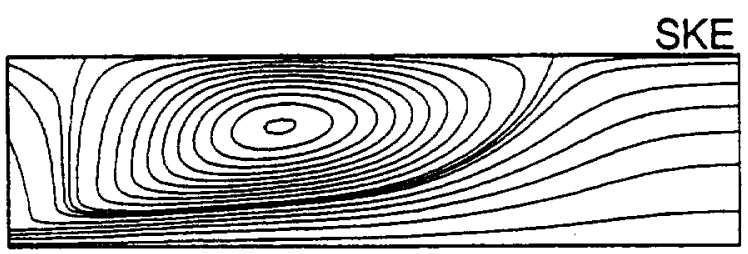

RNG
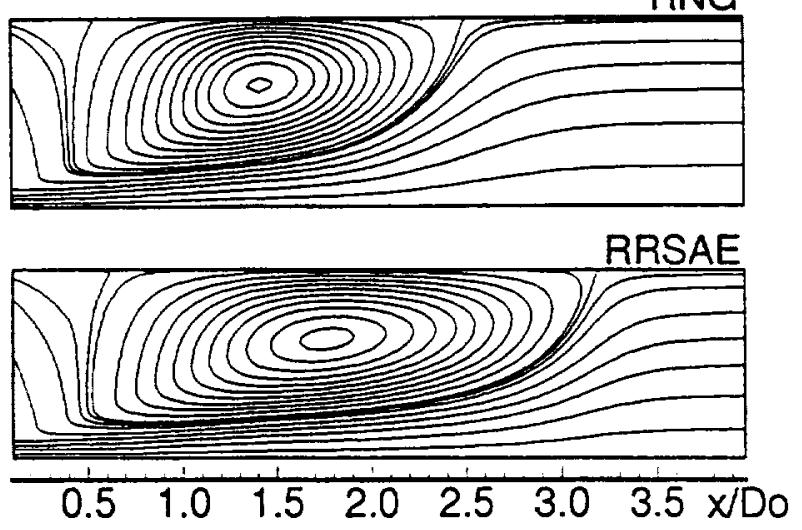

(b) $\mathrm{Ct}=0.152$

Fig.9 Streamlines 


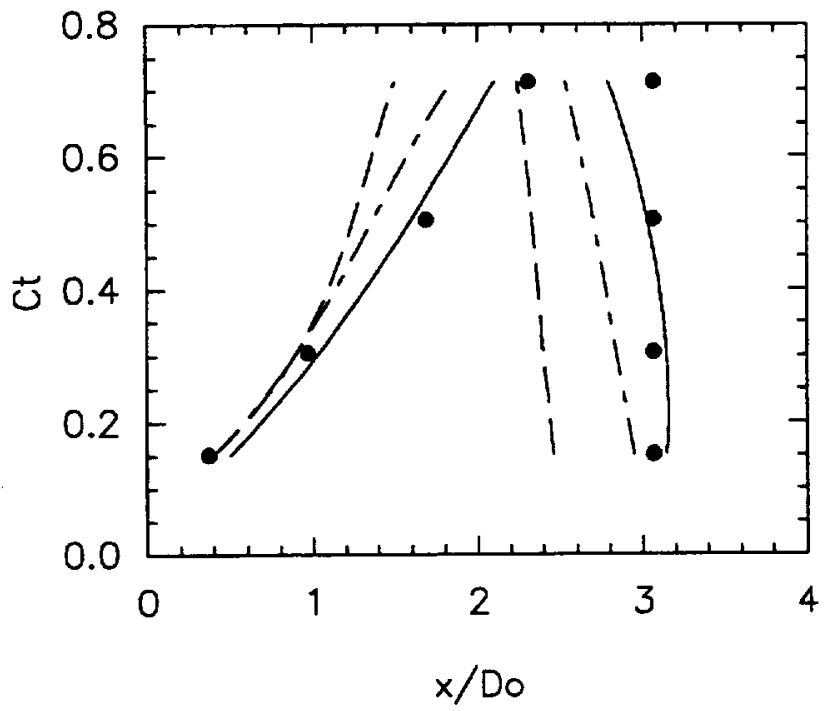

Fig.10 Separation and reattachment points (Notation as in Fig.4)

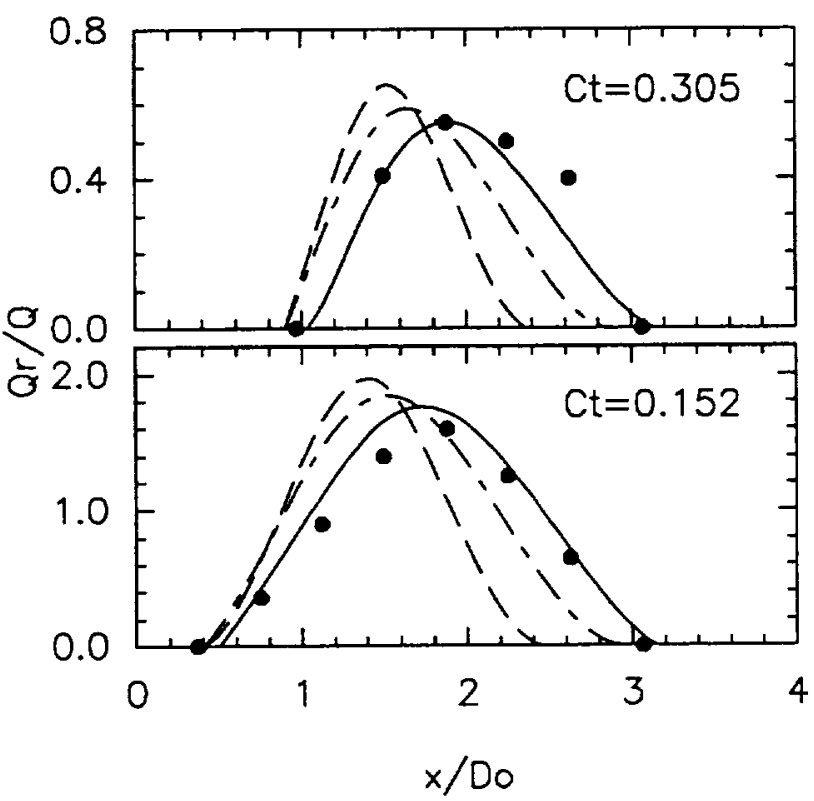

Fig.11 Recirculating flow rate (Notation as in Fig.4)

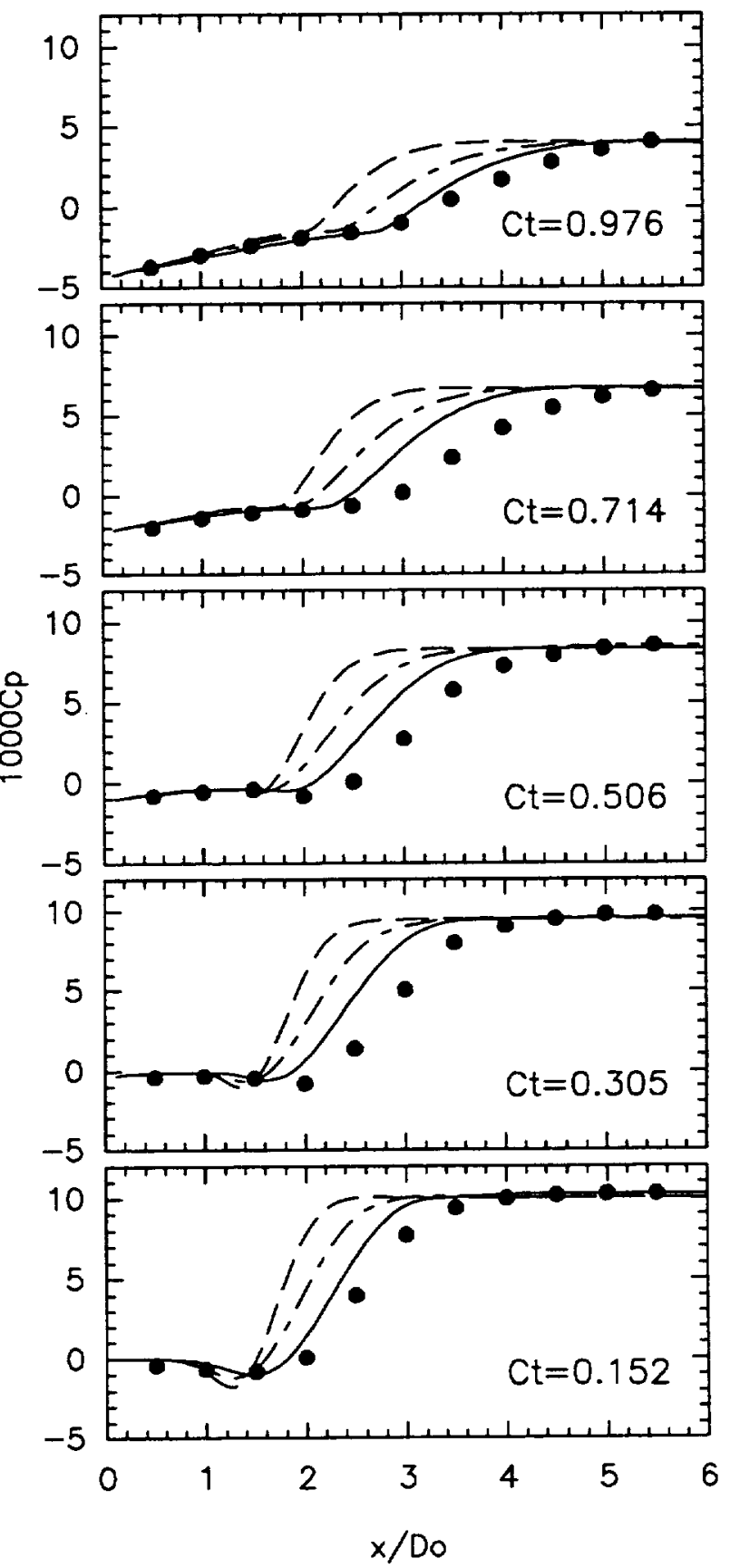

Fig.12 Pressure coefficient at wall (Notation as in Fig.4) 


\begin{tabular}{|c|c|c|c|}
\hline \multicolumn{3}{|c|}{ REPORT DOCUMENTATION PAGE } & $\begin{array}{l}\text { Form Approved } \\
\text { OMB No. } 0704-0188\end{array}$ \\
\hline \multicolumn{4}{|c|}{ 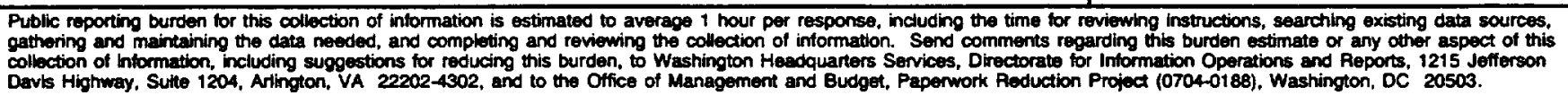 } \\
\hline 1. AGENCY USE ONLY (Leave blank) & $\begin{array}{r}\text { 2. REPOAT DATE } \\
\text { July } 1993\end{array}$ & 3. $\mathrm{F}$ & $\begin{array}{l}\text { ID DATES COVERED } \\
\text { echnical Memorandum }\end{array}$ \\
\hline \multicolumn{3}{|c|}{$\begin{array}{l}\text { 4. ITLE AND SUBTILE } \\
\text { Computation of Confined Coflow Jets With Three Turbulence Model }\end{array}$} & 5. FUNDING NUMBERS \\
\hline \multicolumn{3}{|l|}{$\begin{array}{l}\text { 6. AUTHOR(S) } \\
\text { J. Zhu and T.H. Shih }\end{array}$} & WU-505-90-5K \\
\hline \multicolumn{3}{|c|}{$\begin{array}{l}\text { 7. PERFORMING ORGANEATION NAME(S) AND ADDRESS(ES) } \\
\text { National Aeronautics and Space Administration } \\
\text { Lewis Research Center } \\
\text { Cleveland, Ohio } 44135-3191\end{array}$} & $\begin{array}{l}\text { 8. PERFORMING ORGANIZATION } \\
\text { REPORT NUMBER } \\
\text { E-8191 }\end{array}$ \\
\hline \multicolumn{3}{|c|}{$\begin{array}{l}\text { 9. SPONSORINGMONITORING AGENCY NAME(S) AND ADDRESS(ES) } \\
\text { National Aeronautics and Space Administration } \\
\text { Washington, D.C. } 20546-0001\end{array}$} & $\begin{array}{l}\text { 10. SPONSORINGMNONITORING } \\
\text { AGENCY REPORT NUMBER } \\
\text { NASA TM-106378 } \\
\text { ICOMP-93-40; CMOTT-93-14 } \\
\text { AIAA-93-3120 }\end{array}$ \\
\hline \multicolumn{4}{|c|}{$\begin{array}{l}\text { 11. SUPPLEMENTARY NOTES } \\
\text { Prepared for the 24th AIAA Fluid Dynamics Conference sponsored by the American Institute of Aeronautics and Astronautics, Orlando, Florida, } \\
\text { July 6-9, 1993. J. Zhu and T.H. Shih, Institute for Computational Mechanics in Propulsion and Center for Modeling of Turbulence and Transition, } \\
\text { NASA Lewis Research Center, (work funded under NASA Cooperative Agreement NCC3-233). ICOMP Program Director, Louis A. Povinelli, } \\
\text { (216) 433-5818. }\end{array}$} \\
\hline \multicolumn{3}{|c|}{$\begin{array}{l}\text { 122. DISTRIBUTION/AVAILABILTYY STATEMENT } \\
\text { Unclassified - Unlimited } \\
\text { Subject Category } 34\end{array}$} & 12b. DISTRIBUTION CODE \\
\hline
\end{tabular}

13. ABSTRACT (Meximum 200 words)

A numerical study of confined jets in a cylindrical duct is carried out to examine the performance of two recently proposed turbulence models: an RNG-based K- $\varepsilon$ model and a realizable Reynolds stress algebraic equation model. The former is of the same form as the standard $\mathrm{K}-\varepsilon$ model but has different model coefficients. The latter uses an explicit quadratic stress-strain relationship to model the turbulent stresses and is capable of ensuring the positivity of each turbulent normal stress. The flow considered involves recirculation with unfixed separation and reattachment points and severe adverse pressure gradients, thereby providing a valuable test of the predictive capability of the models for complex flows. Calculations are performed with a finite-volume procedure. Numerical credibility of the solutions is ensured by using second-order accurate differencing schemes and sufficiently fine grids. Calculations with the standard $\mathrm{K}-\varepsilon$ model are also made for comparison. Detailed comparisons with experiments show that the realizable Reynolds stress algebraic equation model consistently works better than does the standard K- $\varepsilon$ model in capturing the essential flow features, while the RNG-based K- $\varepsilon$ model does not seem to give improvements over the standard K- $\varepsilon$ model under the flow conditions considered.

14. SUBJECT TERMS

Confined jets; Turbulence models; Recirculation

\begin{tabular}{|c|c|}
\hline & $\begin{array}{l}\text { 15. NUMBER OF PAGES } \\
12\end{array}$ \\
\hline & $\begin{array}{r}\text { 16. PRICE CODE } \\
\mathrm{AO} 3\end{array}$ \\
\hline $\begin{array}{l}\text { 19. SECUATYY CLASSIFICATION } \\
\text { OF ABSTRACT } \\
\text { Unclassified }\end{array}$ & 20. LMITATION OF ABSTRACT \\
\hline
\end{tabular}

NSN 7540-01-280-5500 